\title{
Correlation Analysis of Real-time Movements Image Processing of Multi Core DSP Based on EM Expectation Algorithm
}

\author{
Guoying Wang \\ Department of Physical Education, Henan College of Traditional Chinese Medicine, Zhengzhou, \\ 450046, China \\ guoying_wang@yeah.net
}

Keywords: Discrete gray, Virtual network, Real time image, Multi core DSP, EM expectation, Correlation

\begin{abstract}
In order to improve the efficiency of image motion correlation analysis, this paper proposes a new algorithm EM expect algorithm in the continuity image motion analysis process. This method uses the discrete gray value of the motion image as a node, and uses the virtual network to optimize the design of node correlation; finally the mathematical model of image correlation analysis is obtained. In order to verify the effectiveness and the reliability of the algorithm, this paper uses the multi-core DSP real-time image processing method to analyze the continuity Wuhu free fight technology and the motion correlation analysis curve is obtained through calculation, which provides the theoretical reference for image cooperative and correlation analysis.
\end{abstract}

\section{Introduction}

In 1977, Dempster, Laird and Rubin (DLR) proposed official EM algorithm, its main role was to use EM to calculate the maximum likelihood estimation in the case of incomplete data [1, 2]. After the proposed EM algorithm, people have studied the nature of EM algorithm and put forward several kinds of improved algorithm, which has been widely used in many fields, such as in mathematical statistics, machine learning, data mining and pattern recognition [3-5]. This paper uses the EM expectation algorithm to design the model of image correlation analysis, and the basic framework is shown in Figure 1.

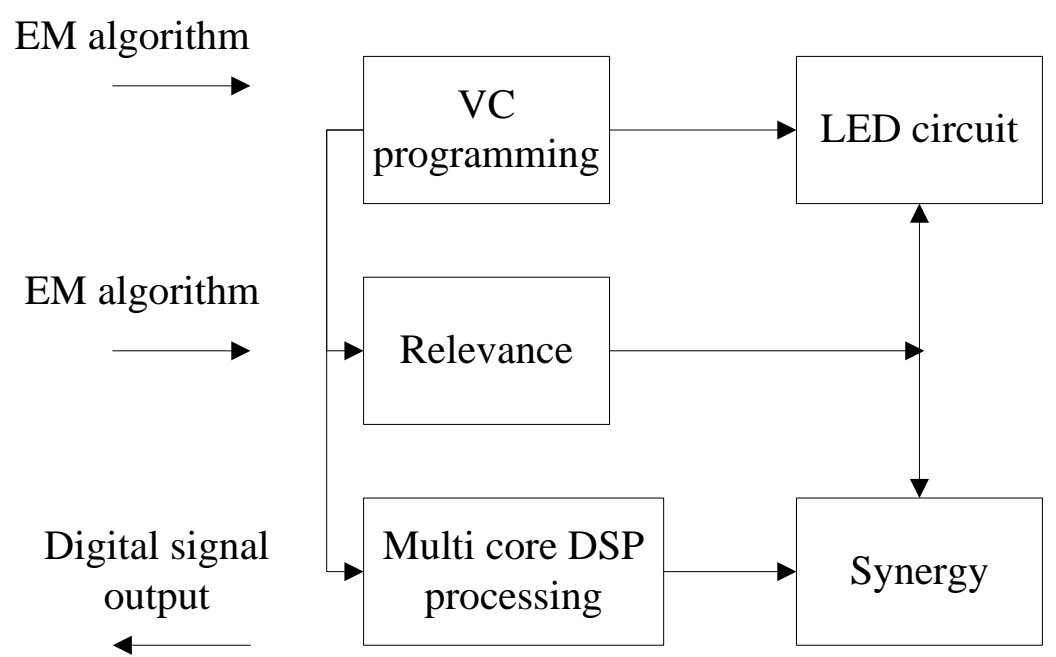

Fig.1: Schematic diagram of computer virtual simulation process

Figure 1 shows schematic diagram of computer image synergetic and correlation analysis. This paper uses EM algorithm to design the image analysis model, and uses the VC algorithm to realize programming methods, and uses the multi core to analyze the continuity motion [6,7]. Finally the image collaboration and correlation analysis results are obtained. 


\section{EM Expectation Mathematical Model and Algorithm Design of Motion Image Coordination}

Motion image collaborative analysis need to use discrete of motion image to achieve. Assuming that network node space matrix of motion image is $T$, and the matrix is symmetric [8]. In order to simplify the calculation, the calculation matrix can be written as below:

$$
T=\left[\begin{array}{cccc}
t_{11} & t_{12} & \cdots & t_{1 n} \\
t_{21} & t_{22} & \cdots & t_{2 n} \\
\cdots & \cdots & \cdots & \cdots \\
t_{n 1} & t_{n 2} & \cdots & t_{n n}
\end{array}\right] .
$$

Among them, $t_{i j}$ is network space adjacent relation between node $i$ and node $j$. If $t_{i}$ is the reference value of position $i$, so the correlation between the nodes is shown in Figure 2.

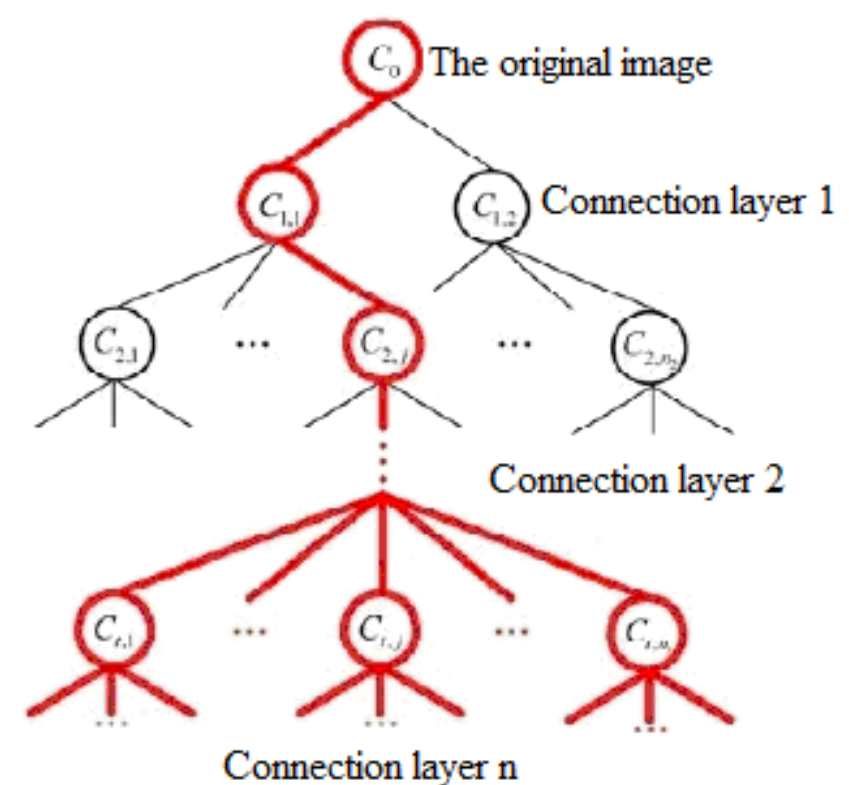

Fig.2: Image analysis of correlation

As shown in Figure 2 it represents schematic structure of image relevance analysis. The basic principle is to find association layer through the original image. This paper adopts the virtual grid node to establish link model, and its expression is as follows:

$$
I=\frac{n \sum_{i=1}^{n} \sum_{j=1}^{n} t_{i j}\left(x_{i}-\bar{x}\right)\left(x_{j}-\bar{x}\right)}{\sum_{i=1}^{n} \sum_{j=1}^{n} t_{i j} \sum_{i=1}^{n}\left(x_{i}-\bar{x}\right)^{2}}=\frac{\sum_{i=1}^{n} \sum_{j \neq i}^{n} t_{i j}\left(x_{i}-\bar{x}\right)\left(x_{j}-\bar{x}\right)}{S^{2} \sum_{i=1}^{n} \sum_{j \neq i}^{n} t_{i j}} .
$$

Among them, $S$ is node distance variance, $x-\bar{x}$ is image correlation, and its formula form is as shown in formula (3).

$$
S^{2}=\frac{1}{n} \sum_{i}\left(x_{i}-\bar{x}\right)^{2}
$$

Among them, $\bar{x}$ is the average value of node distance, and the formula is as follows:

$$
\bar{x}=\frac{1}{n} \sum_{i=1}^{n} x_{i}
$$

This paper uses EM expectation optimization algorithm of image correlation, and the mathematical model of image correlation analysis is as shown follows: 


$$
Y=\frac{(n-1) \sum_{i=1}^{n} \sum_{j=1}^{n} I_{i j}\left(x_{i}-x_{j}\right)^{2}}{2 \sum_{i=1}^{n} \sum_{j=1}^{n} w_{i j} \sum_{i=1}^{n}\left(x_{i}-\bar{x}\right)^{2}} .
$$

In order to realize the EM expectation optimization mathematical model of image correlation, this paper uses VC programming method to design algorithm, and the main procedures are as follows [9-11]:

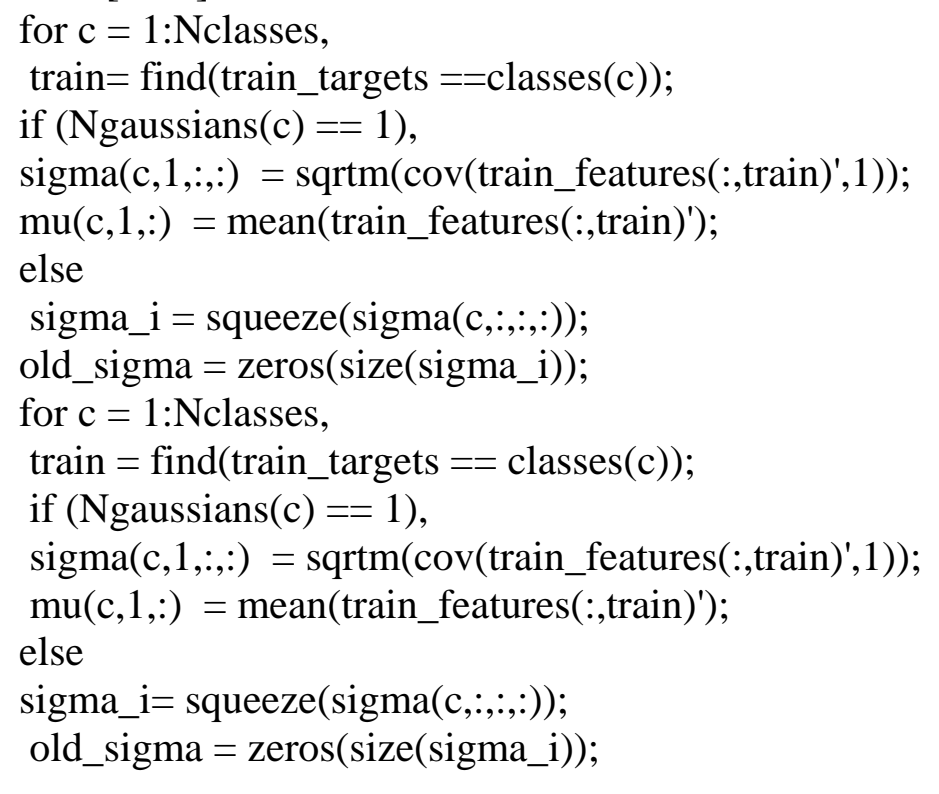

\section{Research on Free Fight Movement Image Correlation Analysis based on EM Expectation}

In order to verify the validity and reliability of EM expectation optimization model designed in second section, this study uses multi-core DSP processing system to calculate the image correlation, and introduces the correlation coupling in the calculation process [12-14]. The principle is as shown in Figure 3.

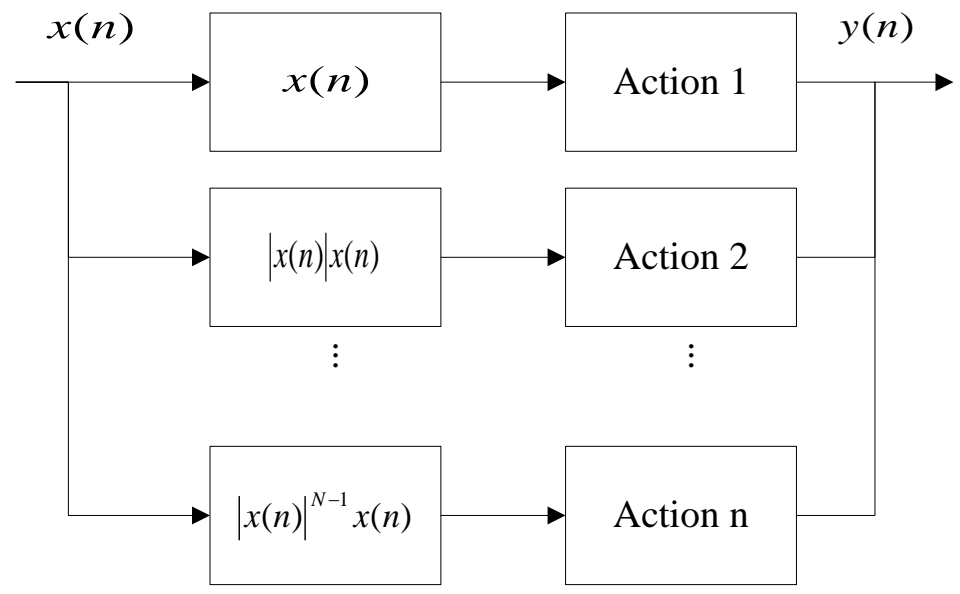

Fig.3: Image coupling analysis diagram

As shown in Figure 3, it represents the coupling image relationship diagram of free fight calculation. It uses EM expectation to analyze the continuity motion, and uses two different models to calculate the correlation [15]. The model is as shown in Figure 4. 


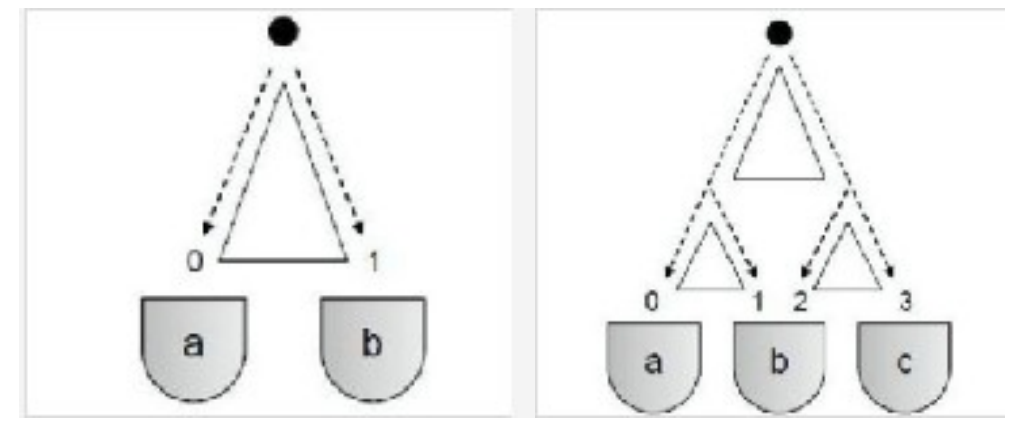

Fig.4: Schematic diagram of simple model and complex model

As shown in Figure 4 it represents the two basic models of free fight movement technology. One is a correlation analysis of single action; the other is the continuous movement analysis of relatively complex. This study selects the second kinds of motion analysis as the research object, as shown in Figure 5.

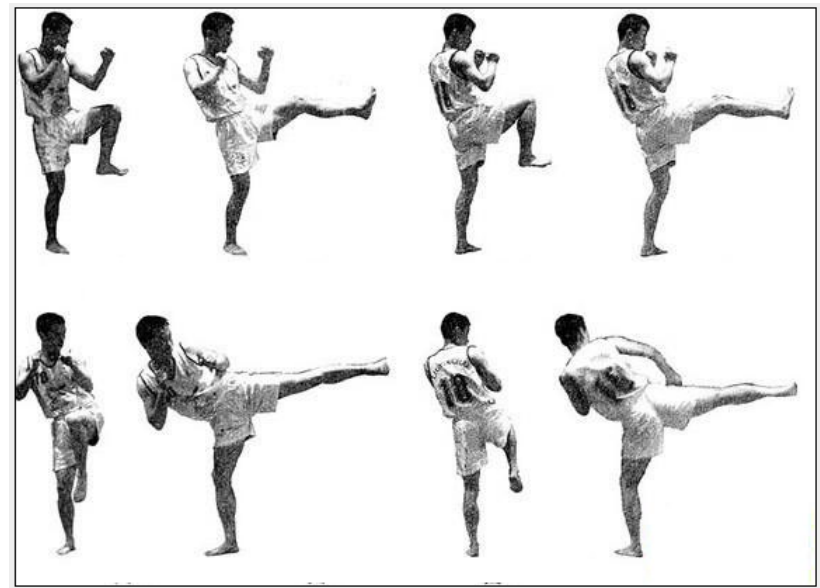

Fig.5: Continuity free fight image analysis

Figure 5 shows the schematic diagram of the continuous free fight movement analysis. As shown in the Figure 5, the action is mainly divided into 8 discrete actions, and it can get synergistic action through the correlation analysis. The processor physical map used is as shown in Figure 6.

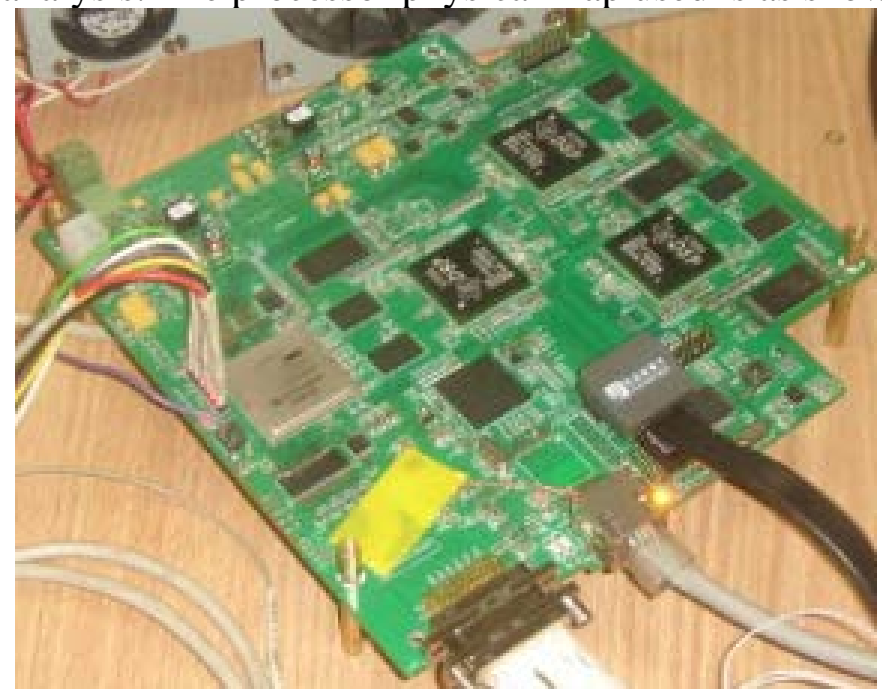

Fig.6: Multi core DSP physical map

As shown in Figure 6, the core of real-time image processing system is DSP processing board. The core circuit of DSP processing board is composed of three DSP, and three DSP is consisting of main control DSP (DSP1\#) and data processing DSP (DSP2\#, DSP3\#). The basic circuit diagram is as shown in Figure 7. 


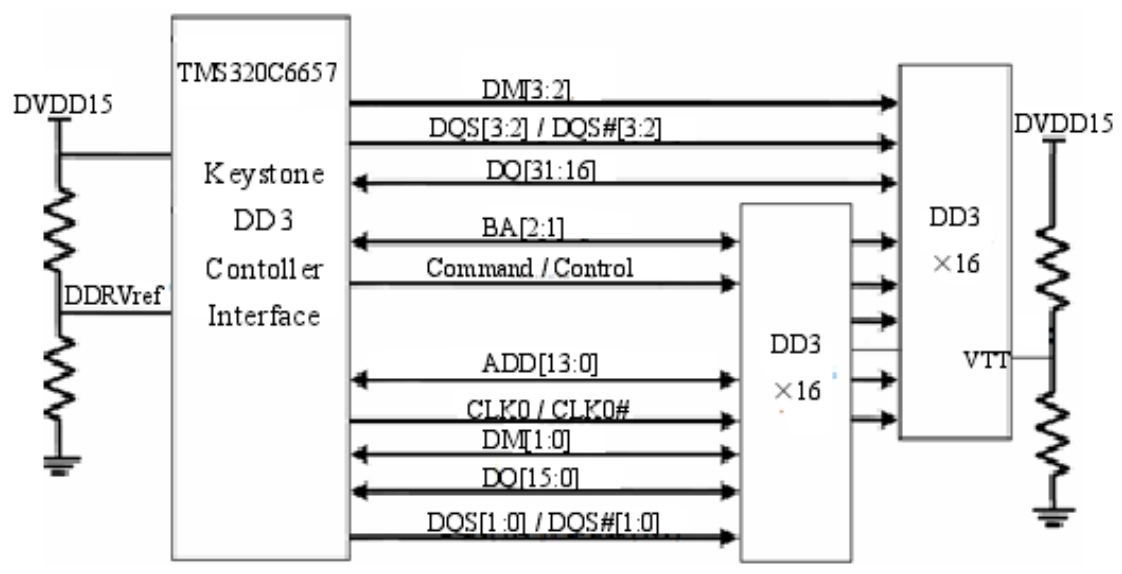

Fig.7: The basic circuit diagram of multi-core processor

As shown in Figure 7, this paper uses multi core TMS320C6657 DDR3 to connect DDR3-1333 SDRAM, supporting DDR3 bus of maximum 32bit, $1333 \mathrm{MHz}$ data rate o and the highest $8 \mathrm{G}$ address space.

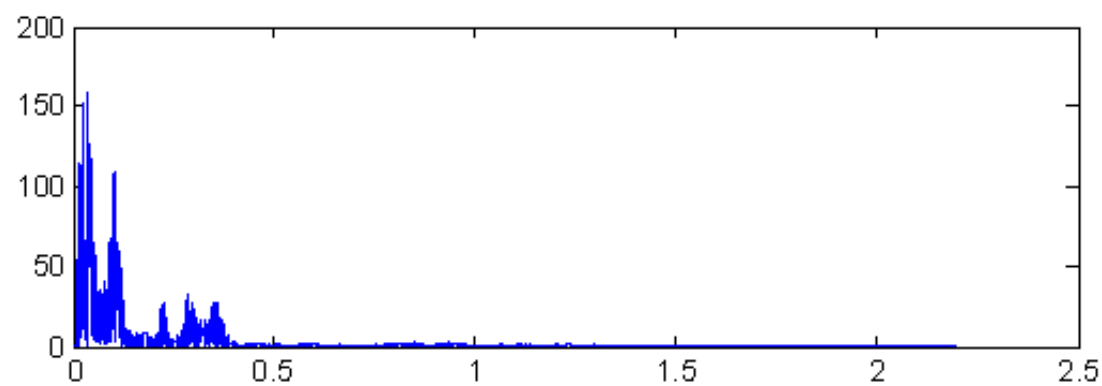

Fig.8: The correlation analysis results of action

As shown in Figure 8, it represents the result curve of using EM expectation algorithm to do correlation analysis on free fight movement. This study processes a set of continuous free fight movement. From the curve it can be seen, the action has high correlation coefficient and strong coordinated action in the initial stage.

\section{Summary}

Based on analyzing theory of continuous action images, according to the EM expectation algorithm, this paper proposes a novel method of image correlation calculation, and uses VC software to design the algorithm, finally uses multi-core DSP processor to process real-time image. In order to verify the effectiveness and reliability of the algorithm, the third part uses single motion model and the continuous action model to design DSP physical map and the basic circuit diagram, finally obtains the correlation analysis results of free fight technology action, which provides a new computer method for the analysis of real time image processing and correlation.

\section{References}

[1] S.Y. Ma. Analysis of Taekwondo starting operation. Contemporary sports science and technology, 2012, 3(6): 56-59.

[2] Y.F. Li, J. Cao. Adolescent tennis athlete's response level. Hubei sports science and technology, 2012, 4(6): 112-114.

[3] X.P. Chen. The factors affecting the reaction rate and the training method. Sports scientific literature review, 2013, 5(11): 42-44.

[4] Y.P. Li, Q.J. Pang. Effect factor of the athlete's reaction velocity. Science and technology promoting the development, 2012, 4(11): 78-80.

[5] J. Miao, X.D. Hang, F. Yu Fang. Sanda coaches training the athletic psychological ability. Free fight, 2012, 4(2): 56-59. 
[6] D.L. Zhang. A comparative study of the twenty-ninth Olympics Sprinters' start reaction time. Journal of Zhaoqing University, 2011, 3(18): 42-45.

[7] Q. Fu. High level fencing visual reaction time characteristics. Journal of Capital Institute of Physical Education, 2012, 4(12): 34-36.

[8] J.M. Zheng, Z.J. Chen. Fast interactive Graph-Cut algorithm with the connectivity constrain. Journal of computer aided design and graphics, 2013, 4(8): 61-64..

[9] S.D. Han, Y. Zhao, W.B. Tao, N. Sang. Fast Graph Cuts image segmentation based on Gauss super pixel method. Journal of automation based on, 2012, 4(20): 101-104.

[10] S.Q. Li, Q. Feng. The Compute Unified Device Architecture parallel graph cut algorithm for liver tumor image segmentation. Journal of Chinese biomedical engineering, 2012, 4(1): 112114.

[11] C. Liu, F.X. Li, Y. Zhang. The objects cut based on graph segmentation and pan shape information. Journal of Computer Aided Design \& computer graphics, 2012, 5(21): 52-56.

[12] Q.P. Sun. The cognitive trait anxiety of elite male Sanda athletes. Shandong sports science and technology, 2012, 4(18): 89-91.

[13] C. Lin, J. Hu, X.Z. Kong. The model and evaluation method of user experience quality. Journal of computer, 2013, 4(2): 45-48.

[14] Y.G. Zeng. Wushu Sanda athletes reaction speed and method. Tsinghua Science and technology, 2012, 3(6): 31-35.

[15] Y. Chang. A comparative reaction time study on adolescent Sanda athletes and adult. Wushu science, 2013, 2(1): 15-18. 